Research Article

\title{
Network Pharmacology and Molecular Docking Analysis on Molecular Targets and Mechanisms of Buyang Huanwu Decoction in the Treatment of Ischemic Stroke
}

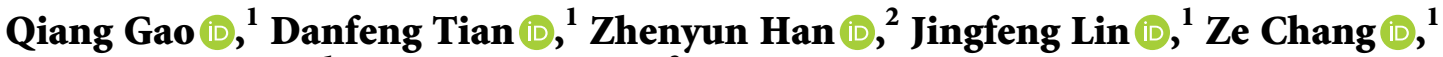 \\ Dandan Zhang $\mathbb{D}^{1}$, and Dayong $M a \mathbb{D}^{3}$ \\ ${ }^{1}$ Beijing University of Chinese Medicine, Beijing 100029, China \\ ${ }^{2}$ Shenzhen Hospital of Beijing University of Chinese Medicine (Longgang), Shenzhen 518172, China \\ ${ }^{3}$ Neurology Department of Dongzhimen Hospital, Beijing University of Chinese Medicine, Beijing 100700, China
}

Correspondence should be addressed to Dayong Ma; ponymdy@163.com

Received 17 September 2020; Revised 1 February 2021; Accepted 9 February 2021; Published 28 February 2021

Academic Editor: Xing Li

Copyright (c) 2021 Qiang Gao et al. This is an open access article distributed under the Creative Commons Attribution License, which permits unrestricted use, distribution, and reproduction in any medium, provided the original work is properly cited.

Background and Objective. With the exact clinical efficacy, Buyang Huanwu decoction (BHD) is a classical prescription for the treatment of ischemic stroke (IS). Here, we aimed to investigate the pharmacological mechanisms of BHD in treating IS using systems biology approaches. Methods. The bioactive components and potential targets of BHD were screened by TCMSP, BATMAN-TCM, ETCM, and SymMap databases. Besides, compounds that failed to find the targets from the above databases were predicted through STITCH, SwissTargetPrediction, and SEA. Moreover, six databases were searched to mine targets of IS. The intersection targets were obtained and analyzed by GO and KEGG enrichment. Furthermore, BHD-IS PPI network, compound-target network, and herb-target-pathway network were constructed by Cytoscape 3.6.0. Finally, AutoDock was used for molecular docking verification. Results. A total of 235 putative targets were obtained from 59 active compounds in BHD. Among them, 62 targets were related to IS. PPI network showed that the top ten key targets were IL6, TNF, VEGFA, AKT1, etc. The enrichment analysis demonstrated candidate BHD targets were more frequently involved in TNF, PI3K-Akt, and NF-kappa B signaling pathway. Network topology analysis showed that Radix Astragali was the main herb in BHD, and the key components were quercetin, beta-sitosterol, kaempferol, stigmasterol, etc. The results of molecular docking showed the active components in BHD had a good binding ability with the key targets. Conclusions. Our study demonstrated that BHD exerted the effect of treating IS by regulating multitargets and multichannels with multicomponents through the method of network pharmacology and molecular docking.

\section{Introduction}

The incidence of ischemic stroke (IS) is particularly high, and survivors have more or less neurological function deficits, which brings about a large burden on society and patients' families. With high mortality and morbidity rate, stroke has been the third most common cause of death following by coronary heart disease and cancer in the world [1]. IS accounts for $70 \%-80 \%$ of all stroke, which is the most common type of stroke in clinic [2]. At present, thrombolysis has been considered as the fastest and most effective treatment for IS, but the clinical effect of thrombolysis therapy is limited due to strict indications, a short time window, high risk of bleeding, and reperfusion injury [3]. A large number of experimental studies and clinical observations have confirmed that Chinese medicine has unique advantages in treating IS. Various empirical prescriptions, single drugs, and active ingredient extracts have shown clear neuroprotective effects on IS [4-6].

Buyang Huanwu decoction (BHD) is a classical prescription for the treatment of IS. This prescription is mainly composed of Huangqi (Radix Astragali), Honghua 
(Carthami flos), Taoren (Persicae Semen), Chishao (Radix Paeoniae Rubra), Danggui (Angelicae sinensis Radix), Chuangxiong (Chuanxiong Rhizoma), and Dilong (Pheretima). Among them, Radix Astragali is the most widely used in the original prescription, which is the monarch drug in BHD. A systematic review and meta-analysis of nineteen RCTs with 1580 individuals showed that BHD could significantly improve the neurological deficit score and the ability of self-care of patients with IS [7]. BHD could promote angiogenesis, attenuate infiltration of natural killer cells, and facilitate neurorehabilitation through an improvement of synaptic plasticity after cerebral ischemia/ reperfusion injury. It could significantly decrease cerebral edema and rat neurological function scores and reduce brain infarct volume [8-12]. Pharmacological researches have shown that Radix Astragali has the function of dilating blood vessels and improving microcirculation, which can significantly increase the brain's ability to withstand periods of severe hypoxia and/or ischemia [13, 14]. Carthami flos, Persicae Semen, Radix Paeoniae Rubra, Angelicae sinensis Radix, and Chuanxiong Rhizoma can effectively improve the microcirculation of the body, significantly inhibit the proliferation of fibrous tissue, and reduce the inflammatory response [15-17].

However, the molecular mechanism of BHD has not been certainly clear. The clinical effect of the decoction is a comprehensive result of the complex biological processes in human body. Network pharmacology, based on system biology and multidirectional pharmacology, integrates the contents of computer biology and network analysis [18]. It explains the integrity and systematization of drug-target-disease interaction from the perspective of multicomponents, multitargets, and multichannels, which is consistent with the holistic view of Chinese medicine [19]. Therefore, it provides a method for the study of multicomponents' mechanisms of Chinese medicine $[20,21]$. In this paper, network pharmacology was used to explore the molecular mechanism of $\mathrm{BHD}$ against IS. The detailed workflow of the study is shown in Figure 1.

\section{Materials and Methods}

2.1. Chemical Ingredients Collection and Active Compounds Screening. Traditional Chinese Medicine Systems Pharmacology Database (TCMSP, http://lsp.nwsuaf.edu.cn) [22], BATMAN-TCM (http://bionet.ncpsb.org/batman-tcm/ index.php/Home/Index/index) [23], ETCM (http://www. tcmip.cn/ETCM/index.php/Home/index/index.html), and SymMap (https://www.symmap.org/) [24] were used to collect the compounds of BHD. Next, ADME analysis was carried out by collecting the main components of $\mathrm{BHD}$ according to the condition parameters $(\mathrm{OB} \geq 30 \%, \mathrm{DL} \geq 0.18$, Caco-2>-0.4) [25]. Through ADME analysis, the potential active components were screened out for further analysis.

2.2. Target Identification. TCMSP, BATMAN-TCM, ETCM, and SymMap databases were used to screen the targets of all the components of BHD. If related targets of components in $\mathrm{BHD}$ could not be found from the above databases, further target prediction was carried out in the STITCH (http:// stitch.embl.de/) [26], SwissTargetPrediction (SwissTargetPrediction, http://www.Swisstargetprediction.ch/) [27], and Similarity Ensemble Approach (SEA, http://sea. bkslab.org/) [28] databases.

Targets related to IS were derived from six public databases, including DisGeNET (http://www.disgenet.org/) [29], OMIM (https://omim.org/) [28], TTD (http://bidd.nus.edu. sg/group/cjttd/), DrugBank (https://www.drugbank.ca/), PharmGKB (https://www.pharmgkb.org/), and MalaCards (https://www.malacards.org/) [30], with keywords "ischemic stroke."

The targets were normalized to the official gene symbols using UniProt database (https://www.uniprot.org/) [31] with the species limited to "Homo sapiens." Finally, the intersection targets of BHD active components and IS were obtained and drawn using a Venn diagram.

2.3. Protein-Protein Interaction Data. String 11.0 (https:// string-db.org/) [32] is a database for storing known and predicted protein interactions, including direct and indirect protein interactions. It scores each protein interaction. A higher score means a higher confidence of protein interaction.

The selected intersection targets were imported into String for protein interaction analysis, and the protein interaction network was obtained with the species limited to "Homo sapiens" and a confidence score $>0.7$. The protein interaction data were imported into Cytoscape 3.6.0 (https:// cytoscape.org/) to construct the PPI network.

2.4. Gene Ontology (GO) and Pathway Enrichment. DAVID (https://david.ncifcrf.gov/) [33] database integrates various types of database resources and uses the improved Fisher precision test algorithm to analyze the enrichment of gene sets. A cutoff $P$ value and false discovery rate (FDR) $<0.05$ were used to indicate statistical significance. GO annotation and KEGG PATHWAY analysis were carried out for the intersection genes. Finally, we could get the pathway maps from KEGG PATHWAY Database (https://www.kegg.jp/) [34].

\subsection{Network Construction and Cluster}

2.5.1. Network Construction. Network construction was performed as follows: (1) BHD-IS PPI network; (2) compound-target network; (3) herb-target-pathway network.

All networks can be constructed via utilizing the network visualization software Cytoscape, which displays network graphically. Cytoscape makes it possible for data integration, analysis, and visualization of complicated networks. In the network diagram, "node" represents the active component and target in BHD, and "edge" represents the relationship between the active component and target. The "degree" parameter, presenting the number of connections between the nodes in the network, was used to evaluate important targets [30]. 


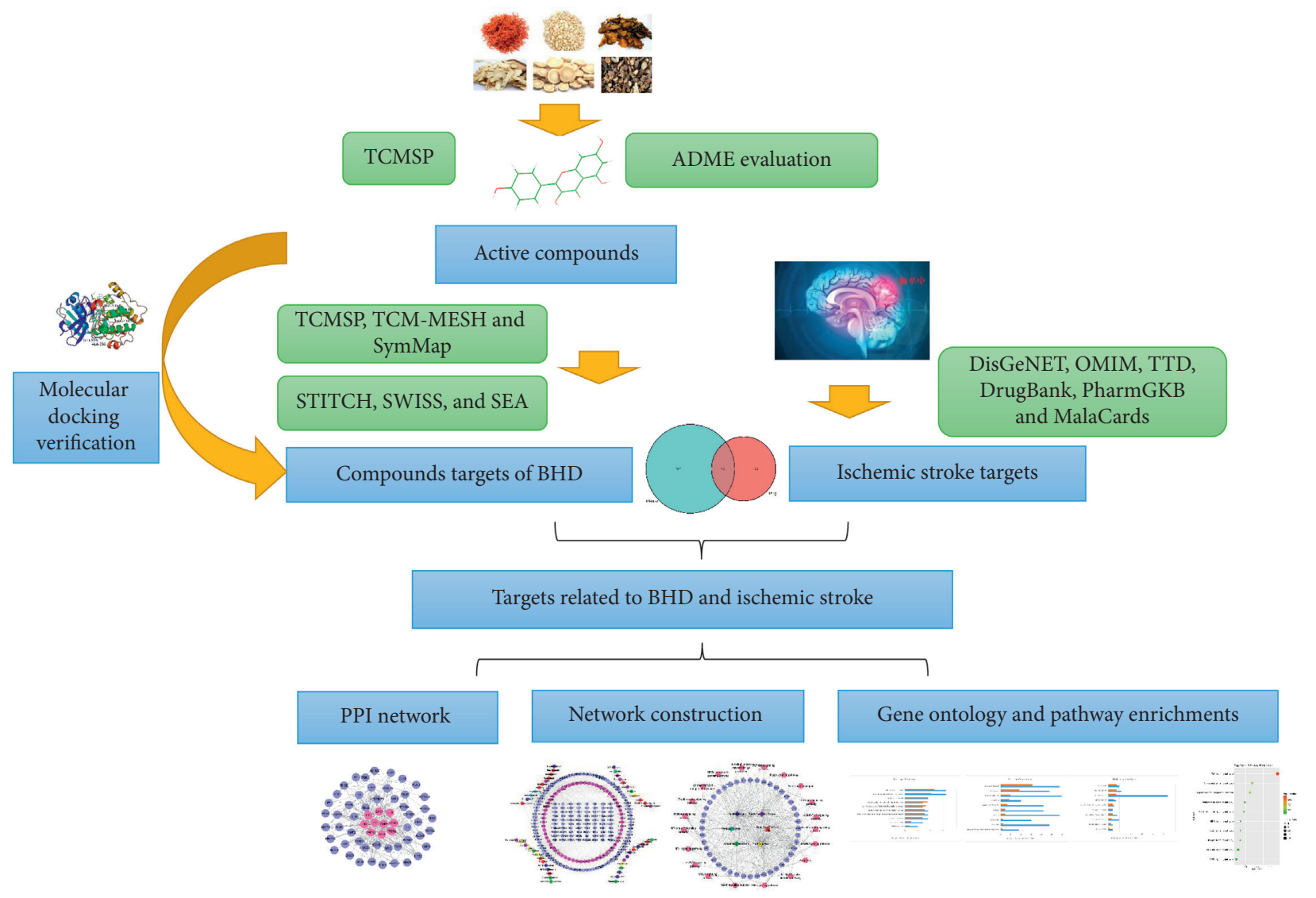

Figure 1: The flowchart of the whole study design.

2.5.2. Cluster of BHD-IS PPI Network. The closely related regions in protein-protein interaction networks are defined as topological modules or clusters. These clusters or functional modules can put nodes of similar or related function together in the same network. By the help of MCODE, a plug-in of Cytoscape, we can get clusters.

2.6. Molecular Docking Verification. To validate the compound-target associations, the AutoDock software (version 4.2) was used to perform the molecular docking program [35]. RCSB PDB (http://www.rcsb.org/) [36] was used to retrieve and download the $3 \mathrm{D}$ structure files of key target proteins. 3D structure files of compounds were downloaded from PubChem (https://pubchem.ncbi.nlm.nih.gov/) [37]. Finally, the AutoDock platform was used for molecular docking verification [38]. The binding energy was calculated to evaluate binding interactions between the compounds and their targets. The binding energy less than " -5 " indicates a good binding interaction between the compound and target [39].

\section{Results}

3.1. Active Compounds. 775 compounds were ultimately reserved: 87 in Radix Astragali, 189 in Chuanxiong Rhizoma, 119 in Radix Paeoniae Rubra, 125 in Angelicae sinensis Radix, 66 in Persicae Semen, 189 in Carthami flos, and 4 in
Pheretima. After ADME screening, 78 potential compounds (OB $\geq 30 \%, D L \geq 0.18$, Caco- $2 \geq 0.4$ ) of seven herbal medicines in BHD were identified, including 16 from Radix Astragali, 6 from Chuanxiong Rhizoma, 20 from Radix Paeoniae Rubra, 2 from Angelicae sinensis Radix, 13 from Persicae Semen, 21 from Carthami flos, and 0 from Pheretima. The details of candidate compounds are described in Table 1. Radix Astragali, Chuanxiong Rhizoma, Radix Paeoniae Rubra, Angelicae sinensis Radix, Persicae Semen, and Carthami flos are simplified as RA, CR, RPR, ASR, PS, and $\mathrm{CF}$, respectively.

3.2. Targets of BHD and IS. As 19 compounds of BHD had no targets in TCMSP, BATMAN-TCM, ETCM, and SymMap, Canonical SMILES of these compounds were found in PubChem. Based on chemical structural similarity, we used databases like STITCH, Swiss TargetPrediction, and SEA, to predict their targets. These compounds were excluded because of the targets score less than $50 \%$ eventually. In brief, 235 targets were adopted in this research.

By means of the six available resources, namely, DisGeNET, OMIM, TTD, DrugBank, PharmGKB and MalaCards databases, we obtained 460 IS-related targets.

Based on targets of the candidate ingredients and IS, intersection targets were got by $\mathrm{R}$ software. 62 intersection genes were found eventually, shown in Figure 2(a). The details of intersection targets are described in Table 2. 
TABLe 1: Active compounds of BHD and their parameters.

\begin{tabular}{|c|c|c|c|c|c|}
\hline Herb & Mol ID & Molecule name & $\begin{array}{l}\text { OB } \\
(\%)\end{array}$ & $\begin{array}{c}\text { Caco- } \\
2\end{array}$ & DL \\
\hline RA & MOL000211 & Mairin & 55.38 & 0.73 & 0.78 \\
\hline RA & MOL000239 & Jaranol & 50.83 & 0.61 & 0.29 \\
\hline RA & MOL000296 & Hederagenin & 36.91 & 1.32 & 0.75 \\
\hline RA & MOL000033 & $\begin{array}{c}\text { (3S,8S,9S,10R,13R,14S,17R)-10,13-Dimethyl-17-[(2R,5S)-5-propan-2-yloctan-2-yl]- } \\
\text { 2,3,4,7,8,9,11,12,14,15,16,17-dodecahydro-1H-cyclopenta[a]phenanthren-3-ol }\end{array}$ & 36.23 & 1.45 & 0.78 \\
\hline RA & MOL000354 & Isorhamnetin & 49.6 & 0.31 & 0.31 \\
\hline RA & MOL000371 & 3,9-Di-O-methylnissolin & 53.74 & 1.18 & 0.48 \\
\hline RA & MOL000378 & 7-O-Methylisomucronulatol & 74.69 & 1.08 & 0.3 \\
\hline RA & MOL000380 & (6aR,11aR)-9,10-Dimethoxy-6a,11a-dihydro-6H-benzofurano [3,2-c] chromen-3-ol & 64.26 & 0.93 & 0.42 \\
\hline RA & MOL000387 & Bifendate & 31.1 & 0.15 & 0.67 \\
\hline RA & MOL000392 & Formononetin & 69.67 & 0.78 & 0.21 \\
\hline RA & MOL000398 & Isoflavanone & 109.99 & 0.53 & 0.3 \\
\hline RA & MOL000417 & Calycosin & 47.75 & 0.52 & 0.24 \\
\hline RA & MOL000422 & Kaempferol & 41.88 & 0.26 & 0.24 \\
\hline RA & MOL000438 & (3R)-3-(2-Hydroxy-3,4-dimethoxyphenyl)chroman-7-ol & 67.67 & 0.96 & 0.26 \\
\hline RA & MOL000442 & 1,7-Dihydroxy-3,9-dimethoxy pterocarpene & 39.05 & 0.89 & 0.48 \\
\hline RA & MOL000098 & Quercetin & 46.43 & 0.05 & 0.28 \\
\hline CR & MOL001494 & Mandenol & 42 & 1.46 & 0.19 \\
\hline CR & MOL002135 & Myricanone & 40.6 & 0.67 & 0.51 \\
\hline CR & MOL002140 & Perlolyrine & 65.95 & 0.88 & 0.27 \\
\hline CR & MOL002151 & Senkyunone & 47.66 & 1.15 & 0.24 \\
\hline CR & MOL002157 & Wallichilide & 42.31 & 0.82 & 0.71 \\
\hline CR & MOL000359 & Sitosterol & 36.91 & 1.32 & 0.75 \\
\hline RPR & MOL001918 & Paeoniflorgenone & 87.59 & -0.09 & 0.37 \\
\hline RPR & MOL001925 & Paeoniflorin_qt & 68.18 & -0.34 & 0.4 \\
\hline RPR & MOL002714 & Baicalein & 33.52 & 0.63 & 0.21 \\
\hline RPR & MOL000358 & Beta-sitosterol & 36.91 & 1.32 & 0.75 \\
\hline RPR & MOL000359 & Sitosterol & 36.91 & 1.32 & 0.75 \\
\hline RPR & MOL004355 & Spinasterol & 42.98 & 1.44 & 0.76 \\
\hline RPR & MOL000449 & Stigmasterol & 43.83 & 1.44 & 0.76 \\
\hline RPR & MOL000492 & $(+)$-Catechin & 54.83 & -0.03 & 0.24 \\
\hline RPR & MOL006992 & $(2 \mathrm{R}, 3 \mathrm{R})-4$-Methoxyl-distylin & 59.98 & 0.17 & 0.3 \\
\hline RPR & MOL006994 & 1-o-Beta-d-glucopyranosyl-8-o-benzoylpaeonisuffrone_qt & 36.01 & -0.03 & 0.3 \\
\hline RPR & MOL006996 & 1-o-Beta-d-glucopyranosylpaeonisuffrone_qt & 65.08 & -0.05 & 0.35 \\
\hline RPR & MOL006999 & Stigmast-7-en-3-ol & 37.42 & 1.32 & 0.75 \\
\hline RPR & MOL007005 & Albiflorin_qt & 48.7 & -0.38 & 0.33 \\
\hline RPR & MOL007008 & 4-Ethyl-paeoniflorin_qt & 56.87 & -0.17 & 0.44 \\
\hline RPR & MOL007012 & 4-o-Methyl-paeoniflorin_qt & 56.7 & 0.4 & 0.43 \\
\hline RPR & MOL007016 & Paeoniflorigenone & 65.33 & -0.13 & 0.37 \\
\hline RPR & MOL007018 & 9-Ethyl-neo-paeoniaflorin A_qt & 64.42 & -0.01 & 0.3 \\
\hline RPR & MOL007022 & Evofolinb & 64.74 & 0 & 0.22 \\
\hline RPR & MOL002883 & Ethyl oleate (NF) & 32.4 & 1.4 & 0.19 \\
\hline RPR & MOL005043 & Campest-5-en-3beta-ol & 37.58 & 1.32 & 0.71 \\
\hline ASR & MOL000358 & Beta-sitosterol & 36.91 & 1.32 & 0.75 \\
\hline ASR & MOL000449 & Stigmasterol & 43.83 & 1.44 & 0.76 \\
\hline PS & MOL001323 & Sitosterol alpha1 & 43.28 & 1.41 & 0.78 \\
\hline PS & MOL001328 & 2,3-Didehydro GA70 & 63.29 & -0.27 & 0.5 \\
\hline PS & MOL001339 & GA119 & 76.36 & -0.12 & 0.49 \\
\hline PS & MOL001340 & GA120 & 84.85 & 0.38 & 0.45 \\
\hline PS & MOL001342 & GA121-isolactone & 72.7 & -0.26 & 0.54 \\
\hline PS & MOL001343 & GA122 & 64.79 & -0.17 & 0.5 \\
\hline PS & MOL001344 & GA122-isolactone & 88.11 & -0.18 & 0.54 \\
\hline PS & MOL001351 & Gibberellin A44 & 101.61 & -0.13 & 0.54 \\
\hline PS & MOL001358 & Gibberellin 7 & 73.8 & -0.18 & 0.5 \\
\hline PS & MOL001371 & Populoside_qt & 108.89 & 0.49 & 0.2 \\
\hline PS & MOL000296 & Hederagenin & 36.91 & 1.32 & 0.75 \\
\hline PS & MOL000358 & Beta-sitosterol & 36.91 & 1.32 & 0.75 \\
\hline PS & MOL000493 & Campesterol & 37.58 & 1.31 & 0.71 \\
\hline $\mathrm{CF}$ & MOL001771 & Poriferast-5-en-3beta-ol & 36.91 & 1.45 & 0.75 \\
\hline
\end{tabular}


TABle 1: Continued.

\begin{tabular}{|c|c|c|c|c|c|}
\hline Herb & Mol ID & Molecule name & $\begin{array}{l}\text { OB } \\
(\%)\end{array}$ & $\begin{array}{c}\text { Caco- } \\
2\end{array}$ & $\mathrm{DL}$ \\
\hline $\mathrm{CF}$ & MOL002680 & Flavoxanthin & 60.41 & 0.97 & 0.56 \\
\hline $\mathrm{CF}$ & MOL002694 & $\begin{array}{l}\text { 4-[(E)-4-(3,5-Dimethoxy-4-oxo-1-cyclohexa-2,5-dienylidene)but-2-enylidene]-2,6- } \\
\text { dimethoxycyclohexa-2,5-dien-1-one }\end{array}$ & 48.47 & 0.81 & 0.36 \\
\hline $\mathrm{CF}$ & MOL002695 & Lignan & 43.32 & 0.42 & 0.65 \\
\hline $\mathrm{CF}$ & MOL002698 & Lupeol-palmitate & 33.98 & 1.52 & 0.32 \\
\hline $\mathrm{CF}$ & MOL002706 & Phytoene & 39.56 & 2.22 & 0.5 \\
\hline $\mathrm{CF}$ & MOL002707 & Phytofluene & 43.18 & 2.29 & 0.5 \\
\hline $\mathrm{CF}$ & MOL002710 & Pyrethrin II & 48.36 & 0.53 & 0.35 \\
\hline $\mathrm{CF}$ & MOL002712 & 6-Hydroxykaempferol & 62.13 & 0.16 & 0.27 \\
\hline $\mathrm{CF}$ & MOL002714 & Baicalein & 33.52 & 0.63 & 0.21 \\
\hline $\mathrm{CF}$ & MOL002717 & qt_Carthamone & 51.03 & -0.31 & 0.2 \\
\hline $\mathrm{CF}$ & MOL002719 & 6-Hydroxynaringenin & 33.23 & 0.27 & 0.24 \\
\hline $\mathrm{CF}$ & MOL002721 & Quercetagetin & 45.01 & -0.06 & 0.31 \\
\hline $\mathrm{CF}$ & MOL002757 & 7,8-Dimethyl-1H-pyrimido[5,6-g]quinoxaline-2,4-dione & 45.75 & 0.06 & 0.19 \\
\hline $\mathrm{CF}$ & MOL002773 & Beta-carotene & 37.18 & 2.25 & 0.58 \\
\hline $\mathrm{CF}$ & MOL000358 & Beta-sitosterol & 36.91 & 1.32 & 0.75 \\
\hline $\mathrm{CF}$ & MOL000422 & Kaempferol & 41.88 & 0.26 & 0.24 \\
\hline $\mathrm{CF}$ & MOL000449 & Stigmasterol & 43.83 & 1.44 & 0.76 \\
\hline $\mathrm{CF}$ & MOL000006 & Luteolin & 36.16 & 0.19 & 0.25 \\
\hline $\mathrm{CF}$ & MOL000953 & CLR & 37.87 & 1.43 & 0.68 \\
\hline $\mathrm{CF}$ & MOL000098 & Quercetin & 46.43 & 0.05 & 0.28 \\
\hline
\end{tabular}

\subsection{Gene Ontology and Pathway Enrichment Analysis}

3.3.1. Gene Ontology. GO analysis of 62 candidate targets for $\mathrm{BHD}$ against IS was performed using the DAVID database to understand the relationship between functional units and their underlying significance in the biological system networks. The results were divided into three parts including biological processes, cellular component, and molecular function, as shown in Figures 3(a)-3(c).

3.3.2. Pathway Enrichment. Through comprehensive analysis, we obtained an integrated IS pathway based on our current knowledge of IS pathogenesis to illuminate the integral role of BHD in treating IS. TOP $10 \mathrm{KEGG}$ signaling pathways of BHD were obtained and constructed based on $P$ value as shown in Figure 3(d).

\subsection{BHD-IS PPI Network Analysis}

3.4.1. BHD-IS PPI Network. 62 intersection targets were imported into the String database, and TSV text showing the interaction relationship was obtained, as shown in Figure 2(b). Then, the network topology analysis was applied by the software of Cytoscape 3.6.0. Importing the TSV text into the Cytoscape software, we could get BHD-IS network, as shown in Figure 2(c). This network contained 59 nodes and 664 edges. In this network, the rose red nodes had higher degrees. The number of those nodes' edges was 36 in IL6, 31 in TNF, 29 in VEGFA, 28 in AKT1, 27 in MMP-9, 26 in IL1B, 23 in MAPK1, 22 in ICAM1, 22 in PTGS2, and 20 in IL10, respectively. This suggested that these genes might be the key or central genes in IS development. Bar graph of all protein nodes degree related to the targets is shown in Figure 2(d). The target proteins in the PPI network were modularized and analyzed by using the plug-in of cluster maker of the software Cytoscape 3.6.0. The results showed that 62 targets were divided into four modules, including 24 in module one, 18 in module two, 11 in module three, and 9 in module four, as shown in Figure 2(e). Functional annotation of the 4 modules is shown in Table 3.

3.4.2. Compound-Target Network Analysis. The active components and their targets were constructed to establish the compound-target network using Cytoscape. This network was composed of 294 nodes (235 compound-target nodes and 59 compound nodes) and 1192 edges. In this network, we could find that one target could be hit by several compounds (central nodes, such as IL6, MMP-9, TNF, AKT1, ICAM1, IL1B, PTGS2, IL-10, VEGFA, and MAPK1), but some were modulated by only one compound in this network. Furthermore, one potential active compound could correspond to multiple targets. Top twelve compounds with high degree were quercetin, beta-sitosterol, kaempferol, stigmasterol, baicalein, luteolin, hederagenin, 7-o-methylis omicron ulatol, formononetin, isorhamnetin, dimethoxy, and myricanone, shown in Table 4 . It could be seen that the neuroprotective mechanism of BHD had the characteristics of multicomponents, multitargets, and multi mechanisms. The compound-target network is shown in Figure 4.

3.4.3. Herb-Target-Pathway Network. By importing 62 targets which overlapped with IS disease genes into DAVID, we could get 20 IS-related pathways. Radix Astragali and Carthami flos had the highest degree, which means that the two herbs might be the main herbs in treating IS. Meanwhile, TNF signaling pathway showed the highest degree, followed 

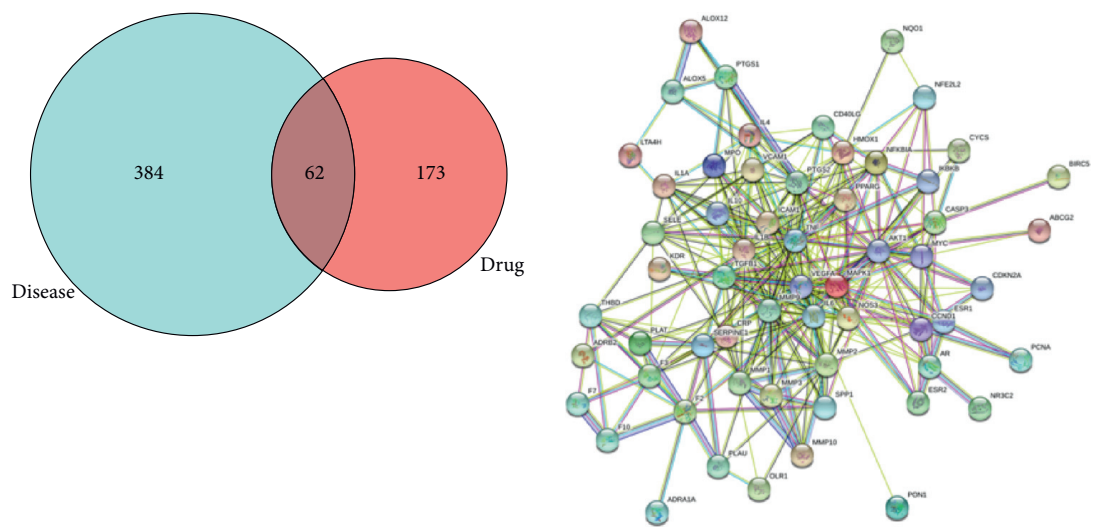

(a)

(b)
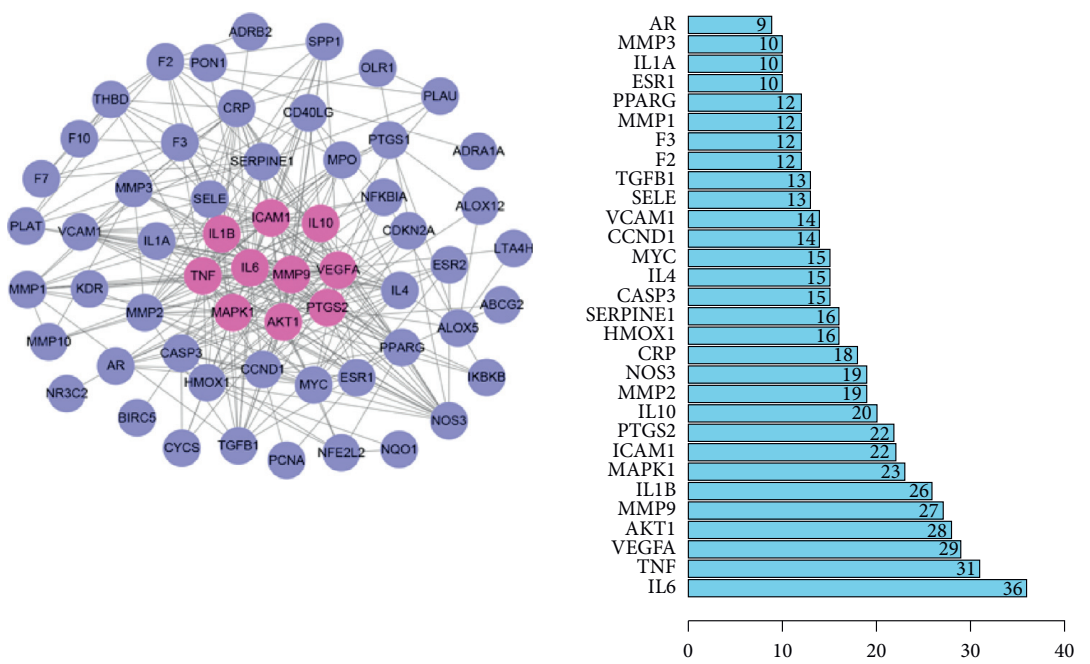

(c)

(d)

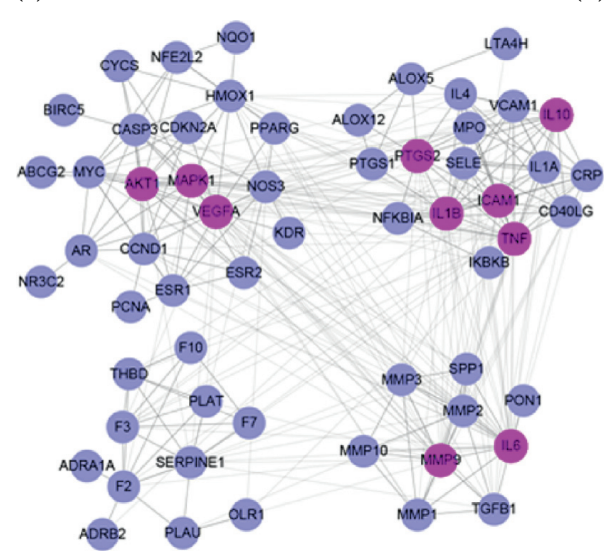

(e)

FIgURE 2: Topological analysis of the target proteins of BHD related to IS. (a) Venn diagram showing shared and unique targets of IS and BHD. (b) The protein-protein interaction (PPI) network diagram constructed by the String database. (c) BHD-IS PPI network constructed by Cytoscape. (d) Bar graph of all protein nodes degree related to the targets. (e) Cluster of PPI network. Lilac and rose red circles stand for 62 intersection targets, and the top ten targets are shown in rose red. 
TABLE 2: Determined target information of BHD related to IS.

\begin{tabular}{|c|c|c|}
\hline UniProt ID & Protein name & Gene name \\
\hline Q9UNQ0 & ABCG2 & ATP-binding cassette sub-family G member 2 \\
\hline P35348 & ADRA1A & Alpha-1A adrenergic receptor \\
\hline P07550 & ADRB2 & Beta-2 adrenergic receptor \\
\hline P31749 & AKT1 & RAC-alpha serine/threonine-protein kinase \\
\hline P18054 & ALOX12 & Arachidonate 12-lipoxygenase, 12S-type \\
\hline P09917 & ALOX5 & Arachidonate 5-lipoxygenase \\
\hline P10275 & AR & Androgen receptor \\
\hline O15392 & BIRC5 & Baculoviral IAP repeat-containing protein 5 \\
\hline $\mathrm{P} 42574$ & CASP3 & Caspase- 3 \\
\hline P24385 & CCND1 & G1/S-specific cyclin-D1 \\
\hline P29965 & CD40LG & CD40 ligand \\
\hline P42771 & CDKN2A & Cyclin-dependent kinase inhibitor $2 \mathrm{~A}$ \\
\hline P02741 & CRP & C-reactive protein \\
\hline P99999 & CYCS & Cytochrome c \\
\hline Q9NRD8 & DUOX2 & Dual oxidase 2 \\
\hline P03372 & ESR1 & Estrogen receptor 1 \\
\hline Q92731 & ESR2 & Estrogen receptor 2 \\
\hline P00742 & F10 & Coagulation factor Xa \\
\hline P00734 & $\mathrm{F} 2$ & Thrombin \\
\hline P13726 & F3 & Tissue factor \\
\hline P08709 & F7 & Coagulation factor VII \\
\hline P42262 & GRIA2 & Glutamate receptor 2 \\
\hline P09601 & HMOX1 & Heme oxygenase 1 \\
\hline P05362 & ICAM1 & Intercellular adhesion molecule 1 \\
\hline O14920 & IKBKB & Inhibitor of nuclear factor kappa-B kinase subunit beta \\
\hline $\mathrm{P} 22301$ & IL10 & Interleukin-10 \\
\hline P01583 & IL1A & Interleukin-1A \\
\hline P01584 & IL1B & Interleukin-1B \\
\hline $\mathrm{P} 05112$ & IL4 & Interleukin-4 \\
\hline P05231 & IL6 & Interleukin-6 \\
\hline P35968 & KDR & Vascular endothelial growth factor receptor 2 \\
\hline P09960 & LTA4H & Leukotriene A-4 hydrolase \\
\hline P11137 & MAP2 & Microtubule-associated protein 2 \\
\hline P28482 & MAPK1 & Mitogen-activated protein kinase 1 \\
\hline P03956 & MMP-1 & Matrix metalloproteinase- 1 \\
\hline P09238 & MMP-10 & Matrix metalloproteinase- 10 \\
\hline P08253 & MMP-2 & Matrix metalloproteinase-2 \\
\hline P08254 & MMP-3 & Matrix metalloproteinase-3 \\
\hline P14780 & MMP-9 & Matrix metalloproteinase- 9 \\
\hline P05164 & MPO & Myeloperoxidase \\
\hline P01106 & MYC & Myc proto-oncogene protein \\
\hline Q16236 & NFE2L2 & Nuclear factor erythroid 2-related factor 2 \\
\hline P25963 & NFKBIA & NF-kappa-B inhibitor alpha \\
\hline P29474 & NOS3 & Nitric oxide synthase, endothelial \\
\hline P15559 & NQO1 & $\mathrm{NAD}(\mathrm{P}) \mathrm{H}$ dehydrogenase [quinone] 1 \\
\hline P08235 & NR3C2 & Mineralocorticoid receptor \\
\hline P78380 & OLR1 & Oxidized low-density lipoprotein receptor 1 \\
\hline P12004 & PCNA & Proliferating cell nuclear antigen \\
\hline P00750 & PLAT & Tissue-type plasminogen activator \\
\hline P00749 & PLAU & Urokinase-type plasminogen activator \\
\hline P27169 & PON1 & Serum paraoxonase/arylesterase 1 \\
\hline P37231 & PPARG & Peroxisome proliferator activated receptor gamma \\
\hline P23219 & PTGS1 & Prostaglandin G/H synthase 1 \\
\hline P35354 & PTGS2 & Prostaglandin G/H synthase 2 \\
\hline P16581 & SELE & E-selectin \\
\hline $\mathrm{P} 05121$ & SERPINE1 & Plasminogen activator inhibitor 1 \\
\hline P10451 & SPP1 & Osteopontin \\
\hline P01137 & TGFB1 & Transforming growth factor beta- 1 \\
\hline P07204 & THBD & Thrombomodulin \\
\hline $\mathrm{P} 01375$ & TNF & Tumor necrosis factor \\
\hline P19320 & VCAM1 & Vascular cell adhesion protein 1 \\
\hline P15692 & VEGFA & Vascular endothelial growth factor A \\
\hline
\end{tabular}




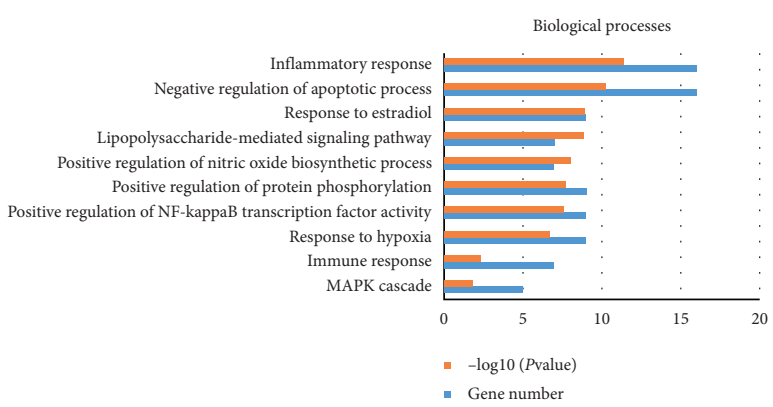

(a)

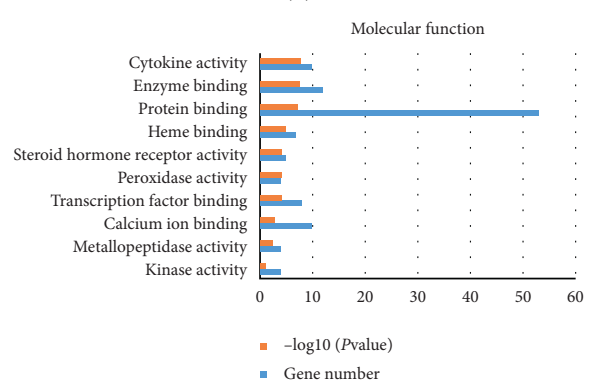

(c)

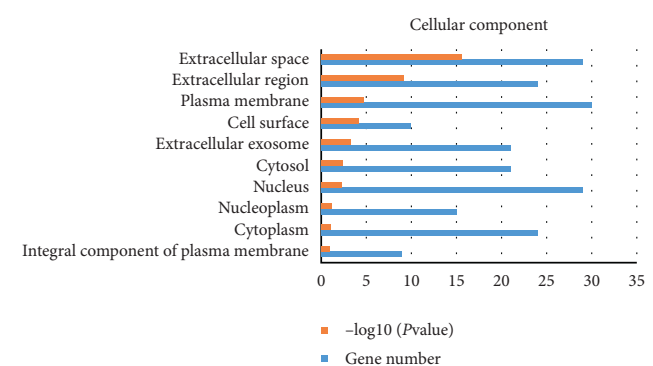

(b)

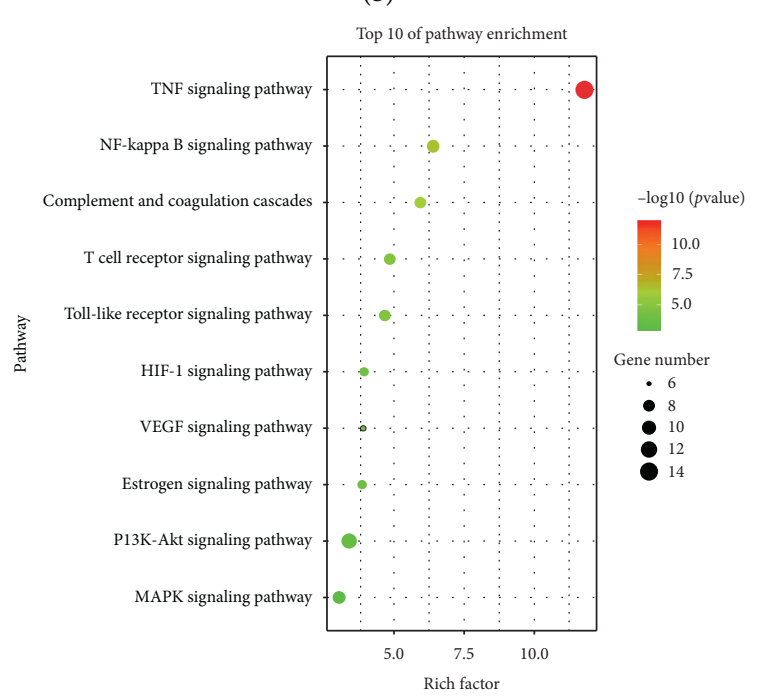

(d)

FIGURE 3: GO and KEGG PATHWAY enrichment analysis for targets of BHD related to IS. (a) Biological processes. (b) Cellular component. (c) Molecular function. (d) Bubble chart of KEGG PATHWAY analysis. The order of importance was ranked by $-\log 10(P$ value $)$ and gene number.

TABLE 3: The main GO enrichment analysis terms of cluster of PPI network.

\begin{tabular}{|c|c|c|c|c|}
\hline Cluster & Term & Genes & $P$ value & FDR \\
\hline 1 & $\begin{array}{c}\text { GO:0045766 positive regulation of } \\
\text { angiogenesis }\end{array}$ & NOS3, KDR, HMOX1, NFE2L2, VEGFA & $9.29 E-06$ & 0.001148 \\
\hline 2 & GO:0006954 inflammatory response & $\begin{array}{l}\text { IL10, CRP, IKBKB, IL1A, CD40LG, IL1B, PTGS2, SELE, } \\
\text { TNF, PTGS1 }\end{array}$ & $2.87 E-11$ & $1.10 E-08$ \\
\hline 3 & GO:0007596 blood coagulation & THBD, F7, F10, PLAU, PLAT, F2, F3 & $3.23 E-10$ & $2.95 E-08$ \\
\hline 4 & $\begin{array}{c}\text { GO:0022617 extracellular matrix } \\
\text { disassembly }\end{array}$ & MMP-1, MMP-2, MMP-3, SPP1, MMP-9, MMP-10 & $9.21 E-11$ & $2.95 E-08$ \\
\hline
\end{tabular}

by PI3K-Akt signaling pathway, MAPK signaling pathway, NF-kappa B signaling pathway, Toll-like receptor signaling pathway, and T cell receptor signaling pathway, respectively. The herb-target-pathway network is shown in Figure 5.

3.5. Molecular Docking Verification. Compound-target interactions with binding energy less than $-5.0 \mathrm{kcal} / \mathrm{mol}$ are shown in Figure 6, including TNF with kaempferol (A), PTGS2 with quercetin (B), MMP-9 with luteolin (C), IL6 with luteolin (D), VEGFA with baicalein (E), IL1B with quercetin (F), ICAM1 with kaempferol (G), MAPK1 with 7O-methylisomucronulatol (H), AKT1 with kaempferol (I), IL-10 with quercetin (J).
3.6. Target Path Analysis. The pathway map of BHD in treating IS was obtained from KEGG PATHWAY Database, as shown in Figure 7. The related pathways were marked in red, and the targets of BHD in treating IS were marked in rose red. The results showed that the main pathways of $\mathrm{BHD}$ in treating IS included TNF signaling pathway, MAPK signaling pathway, NF- $\kappa \mathrm{B}$ signaling pathway, and PI3K/ AKT signaling pathway.

\section{Discussion}

In our study, we found the molecular mechanism of BHD's neuroprotection effect against IS using network 
TABLE 4: The top 12 active components in BHD.

\begin{tabular}{|c|c|c|c|}
\hline Component & Traditional Chinese medicine & Degree & Betweenness centrality \\
\hline Quercetin & Carthami flos & 300 & 0.5468777 \\
\hline Beta-sitosterol & $\begin{array}{c}\text { Radix Paeoniae Rubra } \\
\text { Angelicae sinensis Radix } \\
\text { Persicae Semen } \\
\text { Carthami flos }\end{array}$ & 144 & 0.06546218 \\
\hline Kaempferol & $\begin{array}{l}\text { Hedysarum multijugum Maxim. } \\
\text { Carthami flos }\end{array}$ & 124 & 0.12441394 \\
\hline Stigmasterol & $\begin{array}{c}\text { Radix Paeoniae Rubra } \\
\text { Angelicae sinensis Radix } \\
\text { Carthami flos }\end{array}$ & 87 & 0.05677811 \\
\hline Baicalein & $\begin{array}{c}\text { Radix Paeoniae Rubra } \\
\text { Carthami flos }\end{array}$ & 70 & 0.07380744 \\
\hline Luteolin & Carthami flos & 54 & 0.10769597 \\
\hline Hederagenin & $\begin{array}{c}\text { Hedysarum multijugum Maxim. } \\
\text { Persicae Semen }\end{array}$ & 44 & 0.03690132 \\
\hline 7-O-Methylisomucronulatol & Hedysarum multijugum Maxim. & 43 & 0.07061395 \\
\hline Formononetin & Hedysarum multijugum Maxim. & 36 & 0.06178718 \\
\hline Isorhamnetin & Hedysarum multijugum Maxim. & 34 & 0.04253939 \\
\hline Dimethoxy & Hedysarum multijugum Maxim. & 25 & 0.01472432 \\
\hline Myricanone & Chuanxiong Rhizoma & 23 & 0.01451958 \\
\hline
\end{tabular}

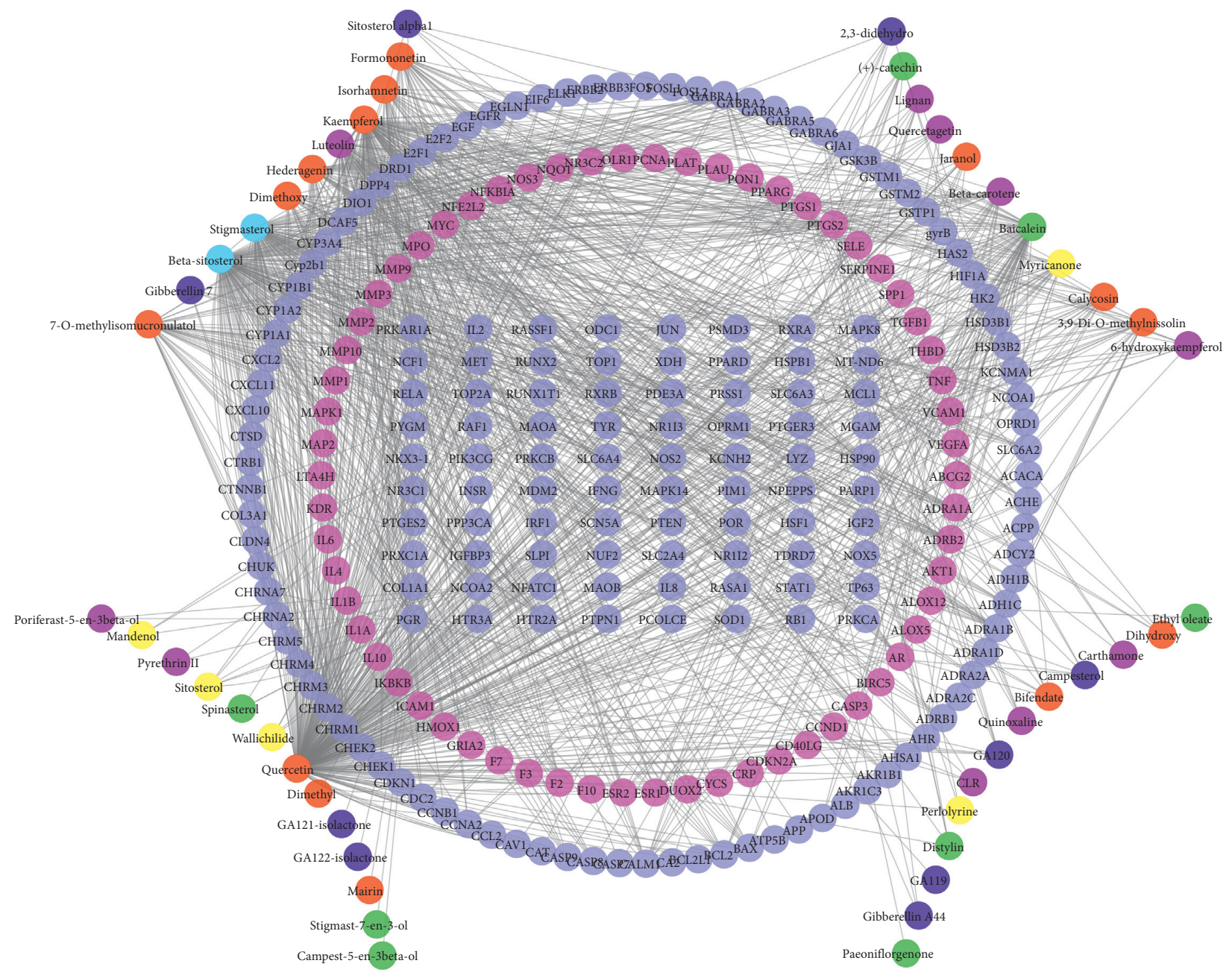

Figure 4: Compound-target network. Light purple and rose red circles stand for the total 235 compound targets, among which the light purple ones represent the 62 intersection targets of BHD and IS. The red, yellow, green, navy blue, light blue, and purple circles stand for active compounds of Radix Astragali, Chuanxiong Rhizoma, Radix Paeoniae Rubra, Persicae Semen, Angelicae sinensis Radix, and Carthami flos. 


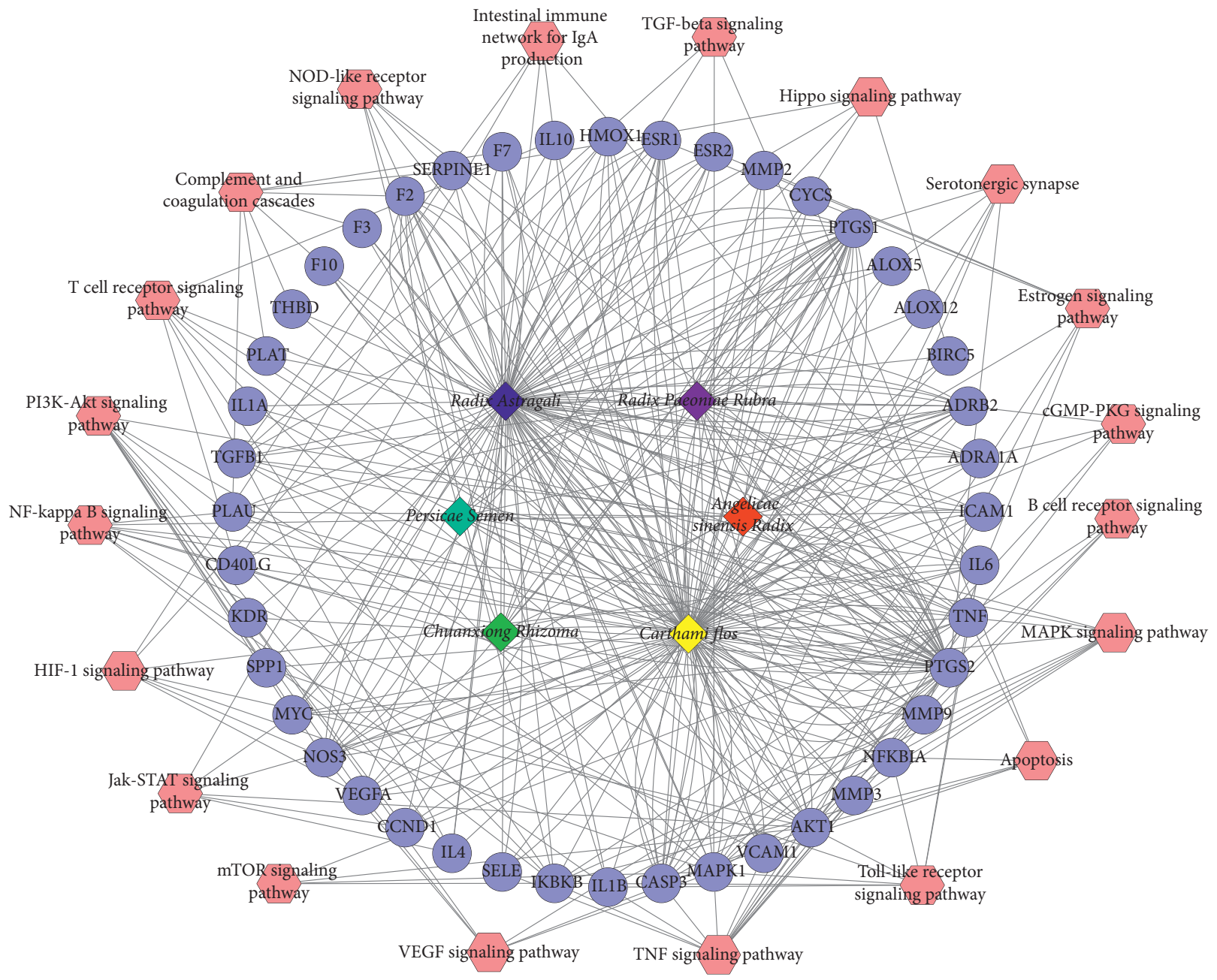

Figure 5: Herb-target-pathway network. The hexagons stand for 20 IS-related pathways and the red, yellow, green, navy blue, light blue, and purple quadrilateral stand for compounds of Radix Astragali, Chuanxiong Rhizoma, Radix Paeoniae Rubra, Persicae Semen, Angelicae sinensis Radix, and Carthami flos, respectively. Besides, lilac circles stand for genes related to BHD.

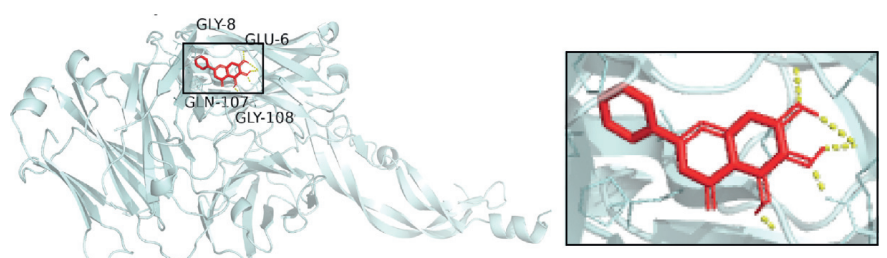

(a)

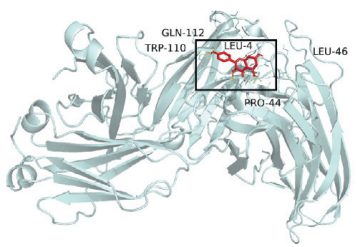

(c)
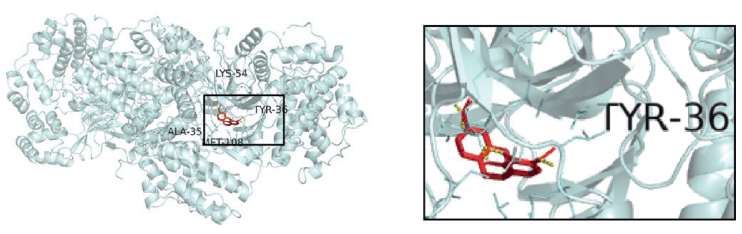

(b)
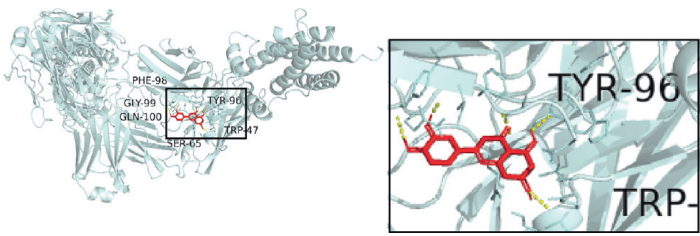

(d)

Figure 6: Continued. 

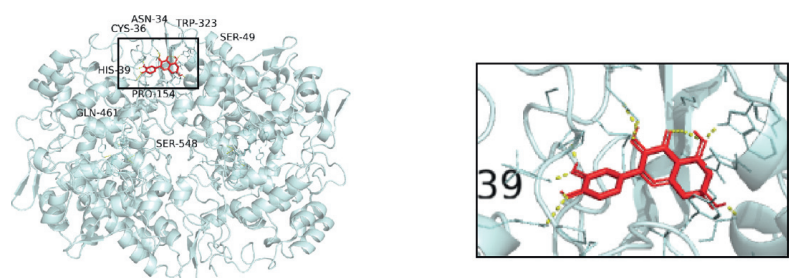

(e)
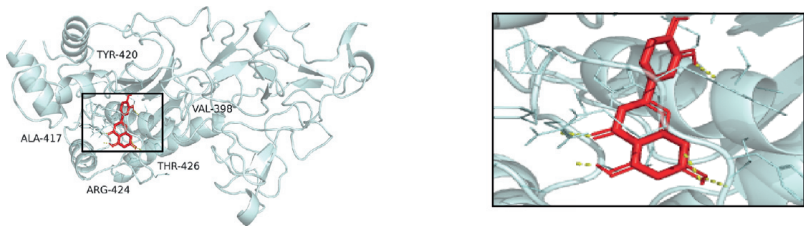

(g)

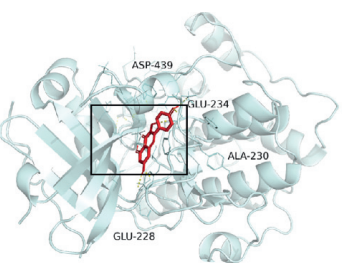

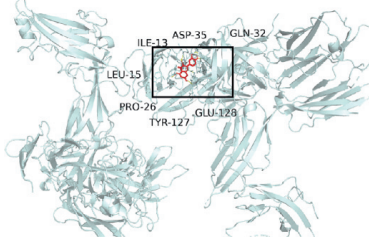

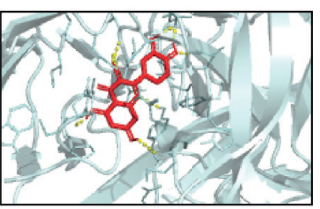

(f)
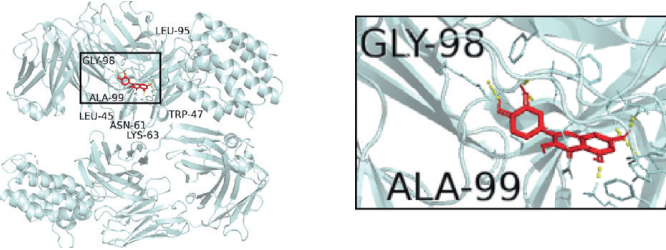

(h)
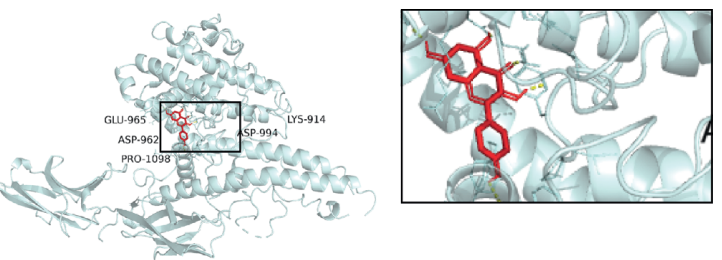

(i)

(j)

FIGURE 6: The conformations of some important compounds and key targets. (a) TNF with kaempferol (binding energy $=-7.67)$. (b) PTGS2 with quercetin (binding energy = -9.29). (c) MMP-9 with luteolin (binding energy = -6.9). (d) IL6 with luteolin (binding energy $=-8.12$ ). (e) VEGFA with baicalein (binding energy $=-6.67$ ). (f) IL1B with quercetin (binding energy $=-8.32$ ). (g) ICAM1 with kaempferol (binding energy $=-8.5$ ). (h) MAPK1 with 7-O-methylisomucronulatol (binding energy $=-6.7)$. (i) AKT1 with kaempferol (binding energy $=-7.96)$. (j) IL-10 with quercetin (binding energy $=-7.55$ ).

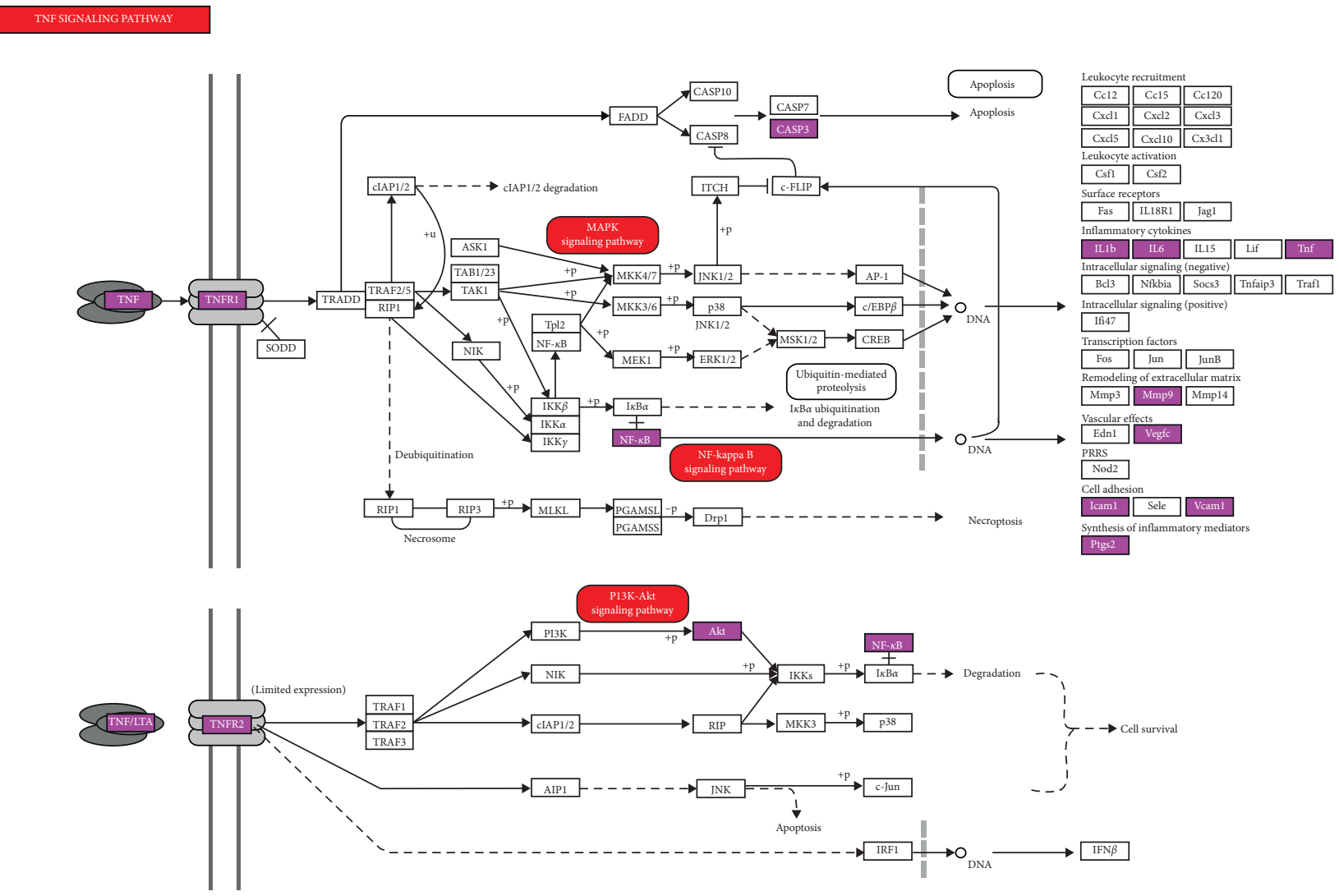

FIGURE 7: Pathway map of BHD against IS. The key targets of BHD in the treatment of IS are shown as rose red in the TNF signal pathway. 
pharmacology strategy. The network pharmacology strategy is helpful to clarify the mechanism of TCM's function from a systematic viewpoint $[40,41]$. Furthermore, this method provides a multidimensional research strategy for various complicated herbal decoctions. At present, the application of network pharmacology to study the mechanism of Chinese medicine has become a research hotspot. In this study, we found that 59 active components of BHD could act on 62 targets related to IS. Further analysis showed that BHD could act on many biological processes of IS and had an influence on the outcome of stroke through TNF, PI3K-Akt, MAPK, and NF-kappa B signaling pathway. It further confirmed that BHD had the characteristics of multicomponents, multichannels, and multitargets.

Core ingredients with the highest degree in compoundtarget network were considered to be responsible for neuroprotection, including quercetin, beta-Sitosterol, kaempferol, stigmasterol, baicalein, luteolin, hederagenin, 7-Omethylisomucronulatol, formononetin, isorhamnetin, Dimethoxy, and myricanone. Six of these components belong to Radix Astragali and Carthami flos. The results of network topology analysis showed that the degrees of Radix Astragali and Carthami flos were the highest in BHD. As the core herb in $\mathrm{BHD}$, the dosage of Radix Astragali is the highest, indicating that the results of network pharmacology are consistent with the clinical application of Chinese medicine. Quercetin and kaempferol are common components of Radix Astragali and Carthami flos. It was found that quercetin could pass through the blood-brain barrier with the highest passage rate [42]. A research showed that quercetin had effects of antioxidant stress and promoting autophagy, which was helpful for the prevention and treatment of stroke [43, 44]. In addition, quercetin could also regulate protein phosphatase $2 \mathrm{~A}$ subunit $\mathrm{B}$ (PP2A) to produce significant neuroprotective effects on rats with cerebral ischemia-reperfusion injury and HT22 cell model of glutamate injury [45]. Lu et al. found that quercetin could inhibit the expression and release of many inflammatory factors such as TNF- $\alpha$, IL- $1 \beta$, and IL6 by reducing the production of $\mathrm{NF}-\kappa \mathrm{B}$ in elderly mice [46]. Kaempferol, a common flavonoid compound, has been widely concerned because of its anti-inflammatory, antioxidant, antibacterial, and antiviral effects. The neuroprotective effect of kaempferol has been confirmed in the acute phase of cerebral infarction [47, 48]. One study confirmed that kaempferol inhibited oxygen-glucose deprivation (OGD) induced cell viability decline, oxidative stress, mitochondrial dysfunction, and apoptosis [49]. These findings suggested that kaempferol might be a promising choice for the intervention of IS. Baicalein is a common component of Carthami flos and Radix Paeoniae Rubra. As an important flavonoid compound, baicalein has many pharmacological effects, such as antioxidant stress, anti-inflammatory, antiexcitatory toxicity, antiapoptosis, stimulating neurogenesis, and promoting the expression of neuroprotective factors [50-52]. Liu et al. found that baicalein had protective effect on transient middle cerebral artery occlusion model rats and could significantly reduce the apoptosis of ischemic penumbra cells around the ischemic infarct of middle cerebral artery occlusion (MCAO) model rats [53]. As an ingredient of Carthami flos, luteolin could downregulate the expression of TLR4, TLR5, NF- $\kappa$ B, and P-P38MAPK, upregulate the expression of p-ERK, and protect cerebral ischemia in rats [54]. Experiments performed in vivo also demonstrated that luteolin reduced the infarct volume. It was suggested that luteolin had a potential in the treatment of IS through inhibiting MMP-9 and activating PI3K/Akt signaling pathway [55]. Beta-sitosterol and stigmasterol are the common components of Carthami flos, Persicae Semen, Radix Paeoniae Rubra, and Angelicae sinensis Radix. They are both sterol compounds, mainly having the functions of reducing blood fat, antioxidation, and anti-inflammation [56].

PPI analysis showed that IL6, TNF, VEGFA, AKT1, MMP-9, IL1B, MAPK1, ICAM1, PTGS2, and IL10 were the top ten targets with high degrees. Followed by cluster of the PPI network, the network could be divided into four modules, which were mainly related to angiogenesis, inflammation, coagulation, and blood-brain barrier. Inflammation plays a critical role in the pathological process of stroke [57]. IL1B, IL10, TNF, IL6, and ICAM1 are closely related to the inflammatory response after stroke, among which IL-10 is an important anti-inflammatory factor, while L1B, TNF, and IL6 are proinflammatory factors. ICAM1 is an important adhesion molecule mediating the adhesion reaction, which plays an important role in stabilizing the interaction between cells and promoting the migration of leukocytes and endothelial cells. Ischemic cascade reaction leads to microglial activation, which will promote the release of proinflammatory cytokines (TNF- $\alpha$, IL1B, and IL6) and anti-inflammatory cytokines (IL10 and TGF- $\beta$ ) [58]. MMP9 is a kind of matrix metalloproteinases (MMPs) closely related to the development of IS, which promotes embryo development, inflammation, atherosclerosis, and other biological functions. Under the stimulation of cerebral ischemia and hypoxia, microglia and astrocytes produce part of MMP-9 under the guidance of inflammatory factors. By hydrolyzing the tight junction protein on the basement membrane of cerebrovascular, the integrity of blood-brain barrier is destroyed [59]. VEGF is a double-edged sword in the development of cerebral infarction. In the hyper acute stage of cerebral infarction, the increase of VEGF concentration increases the permeability of blood-brain barrier, leading to brain edema and aggravate clinical symptoms. In the postinfarction recovery stage, the high content of VEGF is conducive to the establishment of collateral circulation of ischemic focus and penumbra and the damage and repair of neurons [60]. AKT1 is one of serine/threonine-protein kinases (AKT1, AKT2, and AKT3), and it regulates many processes including metabolism, proliferation, cell survival, growth, and angiogenesis. AKT1 gene deletion induces dysfunction of vascular endothelial cells, migration, and survival of vascular smooth muscle cells [61].

Pathway enrichment analysis results showed that TNF signaling pathway, PI3K-Akt signaling pathway, MAPK signaling pathway, and NF-kappa B signaling pathway were the main pathways. TNF signaling pathway is an important inflammatory pathway. As an important cytokine, TNF can induce apoptosis, cell survival, inflammation, immunity, and other intracellular signaling pathways. TNFR1 signal 
transduction can induce the activation of many genes, which are mainly controlled by NF-kappa B and MAPK cascade. In this present study, TNF, IL-1B, MYC, and TGFB1 are potential targets of $\mathrm{BHD}$, suggesting that $\mathrm{BHD}$ plays a neuroprotective role against ischemia-reperfusion injury through TNF signaling pathway. PI3K/Akt signaling pathway is one of the important pathways of cerebral ischemia and neuronal apoptosis. A study found that activating PI3K/ Akt signal pathway could inhibit the apoptosis of nerve and reduce the occurrence of blood-borne brain edema. A series of studies have shown that many Chinese herbal extracts play a protective role in IS through this pathway [62, 63]. Another study found that baicalein also decreased the LC3II/LC3-I ratio and promoted phosphorylation of the PI3K/ Akt/mTOR signaling pathway which implied inhibition of autophagy. The reduction of phosphorylation Akt and glycogen synthase kinase-3beta (GSK3beta) induced by OGD was restored by Baicalein, which was associated with preserved levels of phosphorylation of PTEN [53, 64]. It was reported that baicalein could activate PI3K/AKT pathway, inhibit caspase activation, and reduce cerebral infarct volume in MCAO rats [65]. Besides, formononetin mediated neuroprotection against cerebral ischemia/reperfusion in rats via downregulation of the $\mathrm{Bax} / \mathrm{Bcl}-2$ ratio and upregulation PI3K/Akt signaling pathway [66]. The MAPK signaling pathway may be a therapeutic pathway for stroke [67]. Researches showed that suppressing the NF- $\kappa \mathrm{B}$ and MAPK signaling pathways would downregulate the expression of proinflammatory factors. The MAPK signaling pathway could be a promising candidate for future applications in CNS injury treatment [68]. BHD alleviated pressure overload induced cardiac remodeling by suppressing TGF- $\beta /$ Smads and MAPKs signaling activated fibrosis [69].

However, our research also has some limitations. For example, the accuracy and integrity of existing databases need further verification. Higher quality databases of traditional Chinese medicine and more accurate background network databases are needed. Moreover, the results of network pharmacology need experimental support. The application of network pharmacology in the study of Chinese medicine is just in its start-up step. We need to promote the interdisciplinary researches integrating network science, bioinformatics, computer science, mathematics, and pharmacology in the future.

\section{Conclusion}

In this study, we explored and discussed the characteristic of "multicomponents, multitargets, and multichannels" of BHD-mediated IS treatment through the method of network pharmacology and molecular docking. In the future, we should provide experimental evidence for the neuroprotective effect of BHD against IS according to the results of network pharmacology research.

\section{Data Availability}

All data obtained or analyzed during this study are included within the article.

\section{Conflicts of Interest}

All authors declare no conflicts of interest.

\section{Authors' Contributions}

Qiang Gao contributed to conceptualization and writing original draft. Danfeng Tian contributed to software and data curation. Zhenyun Han contributed to supervision, review, and editing. Jingfeng Lin and Ze Chang contributed to methodology and software. Dandan Zhang contributed to supervision. Dayong Ma contributed to supervision, funding acquisition, and review and editing. Qiang Gao and Danfeng Tian contributed equally to this work.

\section{Acknowledgments}

This work was supported by the National Natural Science Foundation of China (Grant no. 81704049) and "Young Talents" Project of Dongzhimen Hospital of Beijing University of Chinese Medicine (Grant No. DZMYS-201803).

\section{References}

[1] T. Ng, T. Fleming, M. Robinson et al., "Global, regional, and national prevalence of overweight and obesity in children and adults during 1980-2013: a systematic analysis for the global burden of disease study," Lancet, vol. 384, no. 9945, pp. 766-781, 2014.

[2] A. Moretti, F. Ferrari, and R. F. Villa, "Neuroprotection for ischaemic stroke: current status and challenges," Pharmacology \& Therapeutics, vol. 146, pp. 23-34, 2015.

[3] R. Macrez, C. Ali, O. Toutirais et al., "Stroke and the immune system: from pathophysiology to new therapeutic strategies," The Lancet Neurology, vol. 10, no. 5, pp. 471-480, 2011.

[4] H. M. O. Maati, M. Borsotto, F. Chatelain, C. Widmann, M. Lazdunski, and C. Heurteaux, "Activation of ATP-sensitive potassium channels as an element of the neuroprotective effects of the Traditional Chinese Medicine MLC901 against oxygen glucose deprivation," Neuropharmacology, vol. 63, no. 4, pp. 692-700, 2012.

[5] X.-Z. Dong, D.-X. Wang, B.-Y. Yu, P. Liu, and Y. Hu, "KaiXin-San, a traditional Chinese medicine formulation, exerts antidepressive and neuroprotective effects by promoting pCREB upstream pathways," Experimental and Therapeutic Medicine, vol. 12, no. 5, pp. 3308-3314, 2016.

[6] L. F. Liu, J. X. Song, J. H. Lu et al., "Tianma Gouteng Yin, a Traditional Chinese Medicine decoction, exerts neuroprotective effects in animal and cellular models of Parkinson's disease," Scientific Reports, vol. 5, p. 16862, 2015.

[7] C. Hao, F. Wu, J. Shen et al., "Clinical efficacy and safety of Buyang Huanwu decoction for acute ischemic stroke: a systematic review and meta-analysis of 19 randomized controlled trials," Evidence-Based Complementary and Alternative Medicine, vol. 2012, no. 3-4, 10 pages, Article ID 630124, 2012.

[8] Y. She, L. Shao, Y. Zhang et al., "Neuroprotective effect of glycosides in Buyang Huanwu decoction on pyroptosis following cerebral ischemia-reperfusion injury in rats," Journal of Ethnopharmacology, vol. 242, p. 112051, 2019.

[9] X. W. Zheng, C. S. Shan, Q. Q. Xu et al., "Buyang Huanwu decoction targets SIRT1/VEGF pathway to promote angiogenesis after cerebral ischemia/reperfusion injury," Frontiers in Neuroscience, vol. 12, p. 911, 2018. 
[10] B. Dou, W. Zhou, S. Li et al., "Buyang Huanwu decoction attenuates infiltration of natural killer cells and protects against ischemic brain injury," Cellular Physiology and Biochemistry, vol. 50, no. 4, pp. 1286-1300, 2018.

[11] Z.-Z. Chen, X. Gong, Q. Guo, H. Zhao, and L. Wang, "Bu Yang Huan $\mathrm{Wu}$ decoction prevents reperfusion injury following ischemic stroke in rats via inhibition of HIF-1 $\alpha$, VEGF and promotion $\beta$-ENaC expression," Journal of Ethnopharmacology, vol. 228, pp. 70-81, 2019.

[12] R. H. Pan, J. Cai, L. C. Zhan et al., "Buyang Huanwu decoction facilitates neurorehabilitation through an improvement of synaptic plasticity in cerebral ischemic rats," BMC Complementary and Alternative Medicine, vol. 17, no. 1, p. 173, 2017.

[13] M. Ryu, E. H. Kim, M. Chun et al., "Astragali radix elicits antiinflammation via activation of MKP-1, concomitant with attenuation of p38 and Erk," Journal of Ethnopharmacology, vol. 115, no. 2, pp. 184-193, 2008.

[14] H. G. Kim, J. S. Lee, M. K. Choi, J. M. Han, and C. G. Son, "Ethanolic extract of Astragali radix and Salviae radix prohibits oxidative brain injury by psycho-emotional stress in whisker removal rat model," PLoS One, vol. 9, no. 5, p. e98329, 2014.

[15] Y. H. Tu, Y. R. Xue, D. D. Guo, L. N. Sun, and M. L. Guo, "Carthami flos: a review of its ethnopharmacology, pharmacology and clinical applications," Revista Brasileira de Farmacognosia, vol. 25, no. 5, pp. 553-566, 2015.

[16] Z. Yang, H. F. Zhou, P. Zhou, J. H. Yang, and Y. Y. Zhang, "Effects of compatibility of main components from Chuanxiong rhizoma and Astragali radix on hypoxia damaged brain microvascular endothelial cells," Chinese Traditional \& Herbal Drugs, vol. 46, no. 9, pp. 1326-1332, 2015.

[17] R. Chen, P. Wu, Z. Cai et al., "Puerariae lobatae Radix with Chuanxiong rhizoma for treatment of cerebral ischemic stroke by remodeling gut microbiota to regulate the brain-gut barriers," The Journal of Nutritional Biochemistry, vol. 65, pp. 101-114, 2019.

[18] B. Patwardhan, G. Tillu, N. Mehendale, and U. Chandran, "Network pharmacology of ayurveda formulation triphala with special reference to anti-cancer property," Combinatorial Chemistry \& High Throughput Screening, vol. 18, no. 9, pp. 846-854, 2015.

[19] S. Li, B. Zhang, and N. B. Zhang, "Network target for screening synergistic drug combinations with application to traditional Chinese medicine," BMC Systems Biology, vol. 5, p. S10, 2011.

[20] S. Hasan, B. K. Bonde, N. S. Buchan, and M. D. Hall, "Network analysis has diverse roles in drug discovery," Drug Discovery Today, vol. 17, no. 15-16, pp. 869-874, 2012.

[21] Y.-Q. Zhang, X. Mao, Q.-Y. Guo, N. Lin, and S. Li, "Network pharmacology-based approaches capture essence of Chinese herbal medicines," Chinese Herbal Medicines, vol. 8, no. 2, pp. 107-116, 2016.

[22] J. Ru, P. Li, J. Wang et al., "TCMSP: a database of systems pharmacology for drug discovery from herbal medicines," Journal of Cheminformatics, vol. 6, no. 1, p. 13, 2014.

[23] J.-S. You, C.-Y. Li, W. Chen et al., "A network pharmacology-based study on Alzheimer disease prevention and treatment of Qiong Yu Gao,” BioData Mining, vol. 13, p. 2, 2020.

[24] X.-Q. Shi, S.-J. Yue, Y.-P. Tang et al., “A network pharmacology approach to investigate the blood enriching mechanism of Danggui Buxue decoction," Journal of Ethnopharmacology, vol. 235, pp. 227-242, 2019.

[25] W. Yang, Z. Feilong, Y. Kuo et al., "SymMap: an integrative database of traditional Chinese medicine enhanced by symptom mapping," Nucleic Acids Research, vol. 47, no. D1, pp. D1110-D1117, 2018.

[26] M. Zhang, Y. Yuan, W. Zhou et al., "Network pharmacology analysis of Chaihu Lizhong Tang treating non-alcoholic fatty liver disease," Computational Biology and Chemistry, vol. 86, p. 107248, 2020.

[27] D. Gfeller, A. Grosdidier, M. Wirth, A. Daina, O. Michielin, and V. Zoete, "SwissTargetPrediction: a web server for target prediction of bioactive small molecules," Nucleic Acids Research, vol. 42, pp. W32-W38, 2014.

[28] M. J. Keiser, B. L. Roth, B. N. Armbruster, P. Ernsberger, J. J. Irwin, and B. K. Shoichet, "Relating protein pharmacology by ligand chemistry," Nature Biotechnology, vol. 25, no. 2, pp. 197-206, 2007.

[29] N. Queralt-Rosinach, T. Kuhn, C. Chichester, M. Dumontier, F. Sanz, and L. I. Furlong, "Publishing DisGeNET as nanopublications," Semantic Web, vol. 7, no. 5, pp. 519-528, 2016.

[30] J. Zhu, X. Yi, Y. Zhang, Z. Pan, L. Zhong, and P. Huang, "Systems pharmacology-based approach to comparatively study the independent and synergistic mechanisms of Danhong injection and Naoxintong capsule in ischemic stroke treatment," Evidence-Based Complementary and Alternative Medicine, vol. 2019, Article ID 1056708, 17 pages, 2019.

[31] UniProt Consortium, "UniProt: a hub for protein information," Nucleic Acids Research, vol. 43, pp. D204-D212, 2014.

[32] A. Franceschini, D. Szklarczyk, S. Frankild et al., "STRING v9.1: protein-protein interaction networks, with increased coverage and integration," Nucleic Acids Research, vol. 41, pp. D808-D815, 2013.

[33] T. Xu, C. Ma, S. Fan et al., "Systematic understanding of the mechanism of baicalin against ischemic stroke through a network pharmacology approach," Evidence-Based Complementary and Alternative Medicine, vol. 2018, Article ID 2582843, 11 pages, 2018.

[34] L. Shen, W. Chen, B. Zhang, L. Liu, and Y. Cao, "Integrating network pharmacology and bioinformatics analysis to explore the mechanism of Yupingfengsan in treating lung adenocarcinoma," European Journal of Integrative Medicine, vol. 31, p. 100967, 2019.

[35] K. Kaur, P. Kaur, A. Mittal, S. K. Nayak, and G. L. Khatik, "Design and molecular docking studies of novel antimicrobial peptides using autodock molecular docking software," Asian Journal of Pharmaceutical and Clinical Research, vol. 10, no. 16 , p. $28,2017$.

[36] D. S. Goodsell, S. Dutta, C. Zardecki, M. Voigt, H. M. Berman, and S. K. Burley, "The RCSB PDB "molecule of the month": inspiring a molecular view of biology," PLoS Biology, vol. 13, no. 5, p. e1002140, 2015.

[37] E. Vrontaki, G. Melagraki, T. Mavromoustakos, and A. Afantitis, "Searching for anthranilic acid-based thumb pocket $2 \mathrm{HCV}$ NS5B polymerase inhibitors through a combination of molecular docking, 3D-QSAR and virtual screening," Journal of Enzyme Inhibition and Medicinal Chemistry, vol. 31, no. 1, pp. 38-52, 2015.

[38] G. M. Morris, R. Huey, W. Lindstrom et al., "AutoDock4 and AutoDockTools4: automated docking with selective receptor flexibility," Journal of Computational Chemistry, vol. 30, no. 16, pp. 2785-2791, 2009.

[39] T. Gaillard, "Evaluation of AutoDock and AutoDock vina on the CASF-2013 benchmark," Journal of Chemical Information and Modeling, vol. 58, no. 8, pp. 1697-1706, 2018.

[40] C. Chen, H.-L. Li, Y. Yi, H.-J. Fan, and C. Chen, "Network pharmacology-based study on the active substances and mechanism of Nao an capsule in treatment of ischemic 
stroke," European Journal of Integrative Medicine, vol. 31, p. 100976, 2019.

[41] T.-T. Luo, Y. Lu, S.-K. Yan, X. Xiao, X.-L. Rong, and J. Guo, "Network pharmacology in research of Chinese medicine formula: methodology, application and prospective," Chinese Journal of Integrative Medicine, vol. 26, no. 1, pp. 72-80, 2020.

[42] C. Tan, H. Wang, X. Gao et al., "Dynamic changes and prognostic value of gut microbiota-dependent trimethylamine- $\mathrm{N}$-oxide in acute ischemic stroke," Frontiers in $\mathrm{Neu}$ rology, vol. 11, p. 29, 2020.

[43] K. Yamagata, "Polyphenols regulate endothelial functions and reduce the risk of cardiovascular disease," Current Pharmaceutical Design, vol. 25, no. 22, pp. 2443-2458, 2019.

[44] S. Chirumbolo, A. Vella, and G. Bjørklund, "Quercetin might promote autophagy in a middle cerebral artery occlusionmediated ischemia model: comments on Fawad-Ali Shah et al." Neurochemical Research, vol. 44, no. 2, pp. 297-300, 2019.

[45] D.-J. Park, J.-B. Kang, M.-A. Shah, and P.-O. Koh, "Quercetin alleviates the injury-induced decrease of protein phosphatase $2 \mathrm{~A}$ subunit $\mathrm{B}$ in cerebral ischemic animal model and glutamate-exposed HT22 cells," Journal of Veterinary Medical Science, vol. 81, no. 7, pp. 1047-1054, 2019.

[46] J. Lu, D.-M. Wu, Y.-L. Zheng et al., "Quercetin activates AMPactivated protein kinase by reducing $\mathrm{PP} 2 \mathrm{C}$ expression protecting old mouse brain against high cholesterol-induced neurotoxicity," The Journal of Pathology, vol. 222, no. 2, pp. 199-212, 2010.

[47] Y. Lu, C. Chen, L. F. Wang et al., "Neuroprotective effect of kaempferol glycosides against brain injury and neuroinflammation by inhibiting the activation of NF- $\kappa \mathrm{B}$ and STAT3 in transient focal stroke," PLoS One, vol. 8, no. 2, p. e55839, 2013.

[48] B. Wu, H. Luo, X. Zhou et al., "Succinate-induced neuronal mitochondrial fission and hexokinase II malfunction in ischemic stroke: therapeutical effects of kaempferol," Biochimica et Biophysica Acta (BBA)-Molecular Basis of Disease, vol. 1863, no. 9, pp. 2307-2318, 2017.

[49] Y. P. Zhou and G. C. Li, "Kaempferol protects cell damage in in vitro ischemia reperfusion model in rat neuronal PC12 cells," BioMed Research International, vol. 2020, Article ID 2461079, 10 pages, 2020.

[50] W.-Z. Zhao, H.-T. Wang, H.-J. Huang, Y.-L. Lo, and A. M.-Y. Lin, "Neuroprotective effects of baicalein on acrolein-induced neurotoxicity in the nigrostriatal dopaminergic system of rat brain," Molecular Neurobiology, vol. 55, no. 1, pp. 130-137, 2018.

[51] S. Yang, H. Wang, Y. Yang et al., "Baicalein administered in the subacute phase ameliorates ischemia-reperfusion-induced brain injury by reducing neuroinflammation and neuronal damage," Biomedicine \& Pharmacotherapy, vol. 117, p. 109102, 2019.

[52] W. Liang, X. Huang, and W. Chen, "The effects of baicalin and baicalein on cerebral ischemia: a review," Aging and Disease, vol. 8, no. 6, pp. 850-867, 2017.

[53] C. Liu, J. Wu, K. Xu et al., "Neuroprotection by baicalein in ischemic brain injury involves PTEN/AKT pathway," Journal of Neurochemistry, vol. 112, no. 6, pp. 1500-1512, 2010.

[54] H. Qiao, X. Zhang, C. Zhu et al., "Luteolin downregulates TLR4, TLR5, NF- $\kappa$ B and p-p38MAPK expression, upregulates the p-ERK expression, and protects rat brains against focal ischemia," Brain Research, vol. 1448, pp. 71-81, 2012.

[55] S. Luo, H. Li, Z. Mo et al., "Connectivity map identifies luteolin as a treatment option of ischemic stroke by inhibiting
MMP9 and activation of the PI3K/Akt signaling pathway," Experimental \& Molecular Medicine, vol. 51, no. 3, pp. 1-11, 2019.

[56] Y. Liu, C. Kong, L. Gong et al., "The association of post-stroke cognitive impairment and gut microbiota and its corresponding metabolites," Journal of Alzheimer's Disease, vol. 73, no. 4 , pp. 1455-1466, 2020.

[57] C. Angel and H. John, "The harms and benefits of inflammatory and immune responses in vascular disease," Stroke, vol. 37, no. 2, pp. 291-293, 2006.

[58] J. Huang, U. M. Upadhyay, and R. J. Tamargo, "Inflammation in stroke and focal cerebral ischemia," Surgical Neurology, vol. 66, no. 3, pp. 232-245, 2006.

[59] E. Dejonckheere, R. E. Vandenbroucke, and C. Libert, "Matrix metalloproteinases as drug targets in ischemia/reperfusion injury," Drug Discovery Today, vol. 16, no. 17-18, pp. 762-778, 2011.

[60] Y. Bao, L. Qin, E. Kim et al., "CD36 is involved in astrocyte activation and astroglial scar formation," Journal of Cerebral Blood Flow \& Metabolism, vol. 32, no. 8, pp. 1567-1577, 2012.

[61] C. Fernándezhernando, L. József, D. Jenkins, A. D. Lorenzo, and W. C. Sessa, "Absence of Akt1 reduces vascular smooth muscle cell migration and survival and induces features of plaque vulnerability and cardiac dysfunction during atherosclerosis," Arteriosclerosis Thrombosis \& Vascular Biology, vol. 29, no. 12, pp. 2033-2040, 2009.

[62] B. Ou, W. Tao, S. Yang et al., "The antiapoptosis effect of Geum japonicum Thunb. var. chinense extracts on cerebral ischemia reperfusion injury via PI3K/Akt pathway," EvidenceBased Complementary and Alternative Medicine, vol. 2018, no. 1, 13 pages, Article ID 7290170, 2018.

[63] H. Zhang, Y. Song, and C. Feng, "Improvement of cerebral ischemia/reperfusion injury by daucosterol palmitate-induced neuronal apoptosis inhibition via PI3K/Akt/mTOR signaling pathway," Metabolic Brain Disease, vol. 35, no. 6, pp. 1035-1044, 2020.

[64] M. W. Rice, J. D. Pandya, and D. A. Shear, "Gut microbiota as a therapeutic target to ameliorate the biochemical, neuroanatomical, and behavioral effects of traumatic brain injuries," Frontiers in Neurology, vol. 10, p. 875, 2019.

[65] W.-H. Li, Y.-L. Yang, X. Cheng et al., "Baicalein attenuates caspase-independent cells death via inhibiting PARP-1 activation and AIF nuclear translocation in cerebral ischemia/ reperfusion rats," Apoptosis, vol. 25, no. 5-6, pp. 354-369, 2020.

[66] K. Liang, Y. Ye, Y. Wang, J. Zhang, and C. Li, "Formononetin mediates neuroprotection against cerebral ischemia/reperfusion in rats via downregulation of the $\mathrm{Bax} / \mathrm{Bcl}-2$ ratio and upregulation PI3K/Akt signaling pathway," Journal of the Neurological Sciences, vol. 344, no. 1-2, pp. 100-104, 2014.

[67] J. Zhang, Y. Li, C. Wang et al., "Lysophosphatidic acid induces apoptosis of PC12 cells through LPA1 receptor/LPA2 receptor/MAPK signaling pathway," Frontiers in Molecular Neuroscience, vol. 13, p. 16, 2020.

[68] L. Tan, J. Li, Y. Wang, and R. Tan, “Anti-neuroinflammatory effect of alantolactone through the suppression of the NF- $\kappa \mathrm{B}$ and MAPK signaling pathways," Cells, vol. 8, no. 7, p. 739, 2019.

[69] H. Chen, H. Song, X. Liu et al., "Buyanghuanwu Decoction alleviated pressure overload induced cardiac remodeling by suppressing Tgf- $\beta /$ Smads and MAPKs signaling activated fibrosis," Biomedicine \& Pharmacotherapy, vol. 95, pp. 461-468, 2017. 